\title{
DIFERENCIAS SEGÚN GÉNERO Y COLEGIO DE PROCEDENCIA EN LA COMUNICACIÓN INTERPERSONAL
}

\author{
DIFFERENCES BY GENDER AND SCHOOL OF ORIGIN ON INTERPERSONAL \\ COMMUNICATION
}

Carlos Arenas. ${ }^{1}$, Maria Matalinares C., Ornella Raymundo V. Deyvi Baca R.

Universidad Nacional Mayor de San Marcos, Lima, Perú

(RECIBIDO 05/05/2012, ACEPTADO 20/11/2012

\begin{abstract}
RESUMEN
El presente estudio tuvo como objetivo identificar diferencias según género y tipo de colegio en la comunicación interpersonal. La muestra estuvo constituida por 181 estudiantes universitarios de $1^{\circ}$ año, 55 varones y 126 mujeres, el diseño del estudio fue descriptivo comparativo y se utilizó el cuestionario de comunicación interpersonal de Reyes (1996), del cual se extrajo la validez y confiabilidad para el estudio. Los resultados obtenidos demuestran que no existen diferencias estadísticamente significativas según género y colegio de procedencia en la comunicación interpersonal, se resalta la necesidad de realizar estudios en Latinoamerica sobre comunicación interpersonal, asociada a factores sociales y avances tecnológicos.
\end{abstract}

Palabras claves: comunicación interpersonal, estudiantes universitarios.

\begin{abstract}
This study had the objective to identify the differences by gender and kind of school in interpersonal communication, the sample comprised of 181 university students, the study design was descriptive and comparative, the questionnaire was used interpersonal communication of Reyes (1996), extracted the validity and reliability for the study, the results show no statistically significant differences by gender and type of school in interpersonal communication, it highlights the need for studies on interpersonal communication in Latin America related to social and technological.
\end{abstract}

Keywords: interpersonal communication, university students. 


\section{INTRODUCCIÓN}

La comunicación interpersonal tiene una poderosa influencia sobre la conciencia humana, ya que es fundamental para el control y el desarrollo de las relaciones (Aladro, 2009). Ante ello es importante conocer los estudios iniciales sobre comunicación interpersonal, con la finalidad de tener una visión clara de la importancia de esta en la interacción social.

Uno de los primeros estudios sobre la comunicación interpersonal fue el realizado por Mead (como se citó en Garza, 2009). El centro del interés de Mead radicaba en cómo la mente y el concepto de "sí mismo" del individuo (self) emerge de las interacciones con "otros" que le son significativos, a través de un proceso social. Él considera que el sí mismo está constituido a través de la mente, la cognición y el lenguaje (Mead, 1973). Ello implica que las interacciones simbólicas permiten a las personas negociar, manipular, y cambiar, hasta cierto punto, la estructura y la realidad. Por ende, la capacidad humana para usar símbolos significantes es la base para la verdadera comunicación pues el ser humano puede ir más allá del uso del lenguaje gestual. Es así como el interaccionismo simbólico iniciado por Mead tuvo un gran impacto en la psicología, sociología y la comunicación interpersonal.

Heider (1958) acuñó el término Naive Psychology, psicología del sentido común, con ello se inició una corriente llamada “teoría de la atribución”, esta trata sobre las formas en que las personas infieren o atribuyen las causas de la conducta de los otros durante la comunicación interpersonal, siendo afectados por ellos, considerando algunas cuestiones del porqué las personas perciben y evalúan las acciones de las otras personas. Uno de los aportes más importantes de esta teoría es la meta percepción, la cual refiere que el individuo percibe la percepción del otro y probablemente tratará de influir en ella para obtener resultados favorables hacia él (Garza, 2009).

En los siguientes años, la teoría sobre el intercambio social o penetración social, investigada por Kelley y Thibaut (1978), postula la existencia de una interdependencia diádica, la cual refiere que el individuo es capaz de analizar las consecuencias de costo y beneficio de entablar una comunicación, lo que permite o limita su interacción. Por otro lado, la teoría de la congruencia o del sí mismo, ya cobraba valor con la corriente de pensamiento psicológico fenomenológica-existencial, una de las principales contribuciones está en la comprensión empática, la cual si es usada por la persona a través de su comunicación, mejorará sus relaciones con los demás pudiendo tener la capacidad de atender lo que el otro dice, comprendiendo cómo se siente y apreciando el verdadero significado de su mensaje (Rogers, 1974), ante ello se considera a la comunicación como una herramienta para la promoción del desarrollo personal.

La teoría de la autorrevelación, estudiada por Jourard (1971), observa a la comunicación interpersonal bajo la perspectiva de la autorrevelación, maneja el concepto de apertura o transparencia, el primero se entiende por la disposición individual de dejar que el mundo del pensamiento de los otros sea revelado por ellos mismos, y transparencia, es la disposición de la persona para revelarse él mismo a los otros (Jourard, 1971). 
En base a las teorías descritas en Estados Unidos se han generado teorías sobre la comunicación interpersonal, sin embargo se observan pocos estudios realizados en el resto del continente americano. Dentro de las teorías más importantes tenemos a la desarrollada por Kelly (como se citó en Griffin, 2003), quien formuló la teoría constructivista de la comunicación, influenciado por Mead y el interaccionismo simbólico, postula la existencia de los constructos que representan la manera de construir el mundo, ello implica que "la persona le da sentido al mundo a través del sistema de constructos personales". Los constructos son plantillas de la realidad, el mismo que ordena las percepciones (Griffin, 2003). Por otro lado, la teoría de reducción de la incertidumbre desarrollada por Berger (como se citó en Griffin, 2003) retoma el trabajo de Heider sobre la atribución, dando importancia a la asignación de significado a las acciones de los otros, lo cual lo considera como factor dentro de la incertidumbre que surge durante las interacciones personales, pero el principal interés de esta teoría es la reducción de la incertidumbre, lo cual se entiende como la manera de incrementar la predictibilidad acerca del propio comportamiento del sujeto y el de los otros con los que está interactuando (Griffin, 2003). Berger toma como otro factor importante en el desarrollo de una relación y reducción de incertidumbre a la autorevelación propuesta por Jourard, puesto a que ante mayor apertura y transparencia en la comunicación la incertidumbre se reduce, sin embargo está convencido de que la incertidumbre es central en todas las interacciones sociales y la posibilidad de una perfecta comunicación es nula (Berger y Bradac, 1995; Griffin, 2003). Por último, tenemos el modelo de etapas de interacción en las relaciones, desarrollada por Knapp y Vangelisti (1995) describen las características y dinámicas de la comunicación interpersonal tal como se presenta a través de diez etapas que representan el ciclo de vida de una relación, las 5 primeras etapas corresponden a un acercamiento de una relación, las cuales son: iniciación, experimentación, intensificación, integración y unión. Las 5 últimas, corresponden a las etapas de separación en la relación: diferenciación, circunscripción, estancamiento, evitación y terminación. Los autores toman también la autorrevelación desarrollada por Jourard, ya que manifiestan que conforme las etapas van avanzando hacia el acercamiento, la autorrevelación se incrementa y conforme las personas recorren las etapas hacia la separación, esta disminuye. Por otro lado, también consideran los principios planteados por Rogers (como se citó en Garza, 2009), en la teoría de la congruencia y la teoría del intercambio social en cuanto a uso de los costos y beneficios de una relación, como factor que motiva el movimiento de una etapa a otra.

El estudio de la comunicación interpersonal desde la perspectiva latinoamericana, se ocupa del análisis del consumo cultural principalmente de la televisión, la cual abre su interacción en la vida cotidiana, ante ello encontramos a Barbero (1998), quien enfatiza que las teorías hasta el momento ignoran las condiciones y reproducciones del sentido, y redescubre lo popular, que tiene que ver con prácticas tradicionales como rumores, chismes, chistes y relatos populares. Resalta también el papel de la cotidianidad familiar como mediador de los contenidos audiovisuales, propuesta también usada por Orozco (1990). Sin embargo, se puede apreciar 
que en América Latina hay pocos estudios sobre la comunicación interpersonal, como lo refiere Rizo (2008), la comunicación interpersonal no es aún un objeto legitimado en el campo académico de la comunicación latinoamericana, es el trabajo de unos cuantos investigadores los que proveen aportaciones importantes al campo de la comunicación con respecto al estudio de la comunicación interpersonal y temas afines. Por ende, su estudio es de suma importancia considerando las teorías existentes y las variables culturales que caracterizan a Latinoamerica.

Es necesario explorar el nivel de desarrollo de la capacidad de comunicación interpersonal en los individuos de una determina cultura, ya que son las personas quienes construyen sus estrategias de acuerdo con las necesidades que impone la vida social. De acuerdo con cada situación comunicativa, nos vemos precisados a elegir determinadas palabras, a recurrir a ciertas construcciones sintácticas, incluso a modificar el tono y la gestualidad, a mantener la distancia correcta con el otro, distancia lingüística, pero también física (Reyes, 1996).

\section{HIPÓTESIS}

\section{Hipótesis general}

Existen diferencias significativas según género en la comunicación interpersonal. Existen diferencias significativas según el colegio de procedencia en la comunicación interpersonal.

\section{MÉTODO}

\section{Tipo y diseño de investigacion}

En concordancia con las hipótesis formuladas, se empleó el método descriptivo con un diseño transversal comparativo.

\section{Muestra}

El universo poblacional estuvo constituido por alumnos de la UNMSM, siendo la muestra final de 181 sujetos, 55 varones y 126 mujeres.

La muestra total estuvo constituida por alumnos del primer año de las Facultades de Psicología (área A Ciencias de la salud), Educación (Área B Humanidades) y derecho (Área C Ciencias sociales).

\section{Técnicas e Instrumento}

Para el presente estudio se utilizó la técnica psicométrica. Se utilizó el cuestionario de comunicación interpersonal elaborado por Reyes (1996) con 20 preguntas con opciones múltiples, cuyo objetivo es identificar la dinámica comunicativa en las interacciones sociales, en el diario vivir de las conversaciones. Los resultados indican el nivel de desarrollo en la comunicación interpersonal del sujeto, considerando tres niveles: comunicado novato, comunicador dispuesto y comunicador natural. 
En la presente investigación se realizó un análisis psicométrico del cuestionario, en cuanto a la validez de contenido el cuestionario fue revisado por expertos en la temática, quienes confirmaron la pertinencia de los ítems, asimismo, se obtuvo una confiabilidad de 0.79 , según el procedimiento dado por la fórmula $\mathrm{Kr} 20$ o Kuder Richardson, el cual está en el límite de muy alto, es decir una confiabilidad por encima del nivel de aceptable.

\section{RESULTADOS}

\section{Análisis descriptivo}

Tabla 1

Estadísticas descriptivas del cuestionario de comunicación interpersonal

\begin{tabular}{lc}
\hline Estadística descriptiva & \\
\hline Media & 55.5 \\
Error típico & 0,63 \\
Desviación estándar & 8,43 \\
Varianza de la muestra & 71,07 \\
Curtosis & 0.90 \\
Coeficiente de asimetría & $-0,66$ \\
\hline
\end{tabular}

En la Tabla 1, se aprecia que el valor de la media aritmética es 55.5, lo cual indica que los hábitos de comunicación se ubican en el nivel de comunicador dispuesto en los estudiantes de la UNMSM.

Tabla 2

Frecuencias y porcentajes del perfil de comunicación interpersonal

\begin{tabular}{lcc}
\hline Perfil & Frecuencia & $\%$ \\
\hline Comunicador novato & 13 & 7.5 \\
\hline Comunicador dispuesto & 148 & 81.5 \\
\hline Comunicador natural & 20 & 11 \\
\hline Total & 181 & 100 \\
\hline
\end{tabular}

Según lo observado en la Tabla 2, son 13 sujetos que se ubican con el perfil de comunicadores novatos, equivalen a un $7.5 \%, 148$ alumnos se encuentran el perfil de comunicadores dispuestos equivalen al $81.5 \%$ de la muestra y 20 alumnos se ubican en el perfil de comunicadores naturales correspondiente al $11 \%$ de la muestra total. 
Tabla 3 Distribución porcentual de respuestas por pregunta del cuestionario de comunicación interpersonal

\begin{tabular}{lllllllllllllllllllll}
\hline Ítem & 1 & 2 & 3 & 4 & 5 & 6 & 7 & 8 & 9 & 10 & 11 & 12 & 13 & 14 & 15 & 16 & 17 & 18 & 19 & 20 \\
Siempre & 8 & 8 & 12 & 10 & 11 & 12 & 9 & 6 & 20 & 13 & 15 & 6 & 11 & 11 & 27 & 6 & 14 & 11 & 4 & 4 \\
A veces & 82 & 72 & 70 & 73 & 62 & 62 & 79 & 80 & 70 & 69 & 74 & 76 & 69 & 79 & 57 & 78 & 70 & 74 & 72 & 80 \\
& & & & & & & & & & & & & & & & & & & & \\
Nunca & 10 & 20 & 18 & 18 & 37 & 27 & 12 & 15 & 10 & 18 & 11 & 18 & 20 & 10 & 16 & 16 & 16 & 14 & 24 & 16 \\
\hline
\end{tabular}

En la Tabla 3 se aprecia que los alumnos tienden a dar una respuesta media, "a veces", lo cual revelaría habilidades de comunicación a nivel medio, es decir, comunicador novato a dispuesto. A continuación se detallan las preguntas con su porcentaje de respuesta; 1.- el $82 \%$ de los evaluados a veces interrumpe con frecuencia a su interlocutor, 2.- el $80 \%$ refiere que siempre o a veces evita la mirada en el hablante, 3.- el $82 \%$ a veces o siempre prefiere ver TV o leer prensa que dialogar con sus familiares o amigos, 4.- el $84 \%$ a veces o siempre comenta un suceso repetidamente con la misma persona, 5.- el $73 \%$ a veces o siempre ignora cuál es el momento oportuno para terminar una conversación, 6.- el $74 \%$ a veces o siempre se disgusta cuando los demás no entienden lo que dice, 7.- el $88 \%$ a veces o siempre se siente nervioso cuando habla frente a personas extrañas, 8.- el $86 \%$ a veces o siempre es el que habla durante todo el tiempo durante la conversación, 9.- el $90 \%$ a veces o siempre evita las conversaciones, 10.- el $82 \%$ a veces o siempre utiliza palabras groseras cuando habla, 11.- el $89 \%$ a veces o siempre interrumpe las conversaciones ajenas, 12.- el $82 \%$ a veces o siempre se reserva el uso de anécdotas en la comunicación, 13.- el $80 \%$ a veces o siempre le es indiferente el silencio en una conversación, 14.- el $90 \%$ a veces o siempre habla con rodeos, 15.- el $84 \%$ a veces o siempre habla solo, 16.- el $84 \%$ a veces o siempre su atención se dispersa en una charla, 17.- el $84 \%$ a veces o siempre se le dificulta relacionarse con las personas fácilmente, 18.- el $85 \%$ a veces o siempre comienza un tema sin terminarlo, 19.- el $76 \%$ a veces o siempre mueve mucho las manos cuando quiere expresar algo, y por último 20.- el $84 \%$ a veces o siempre cuando conversa se olvida las palabras.

Tabla 4

Resultados de la comparación de la comunicación interpersonal según el género

\begin{tabular}{|c|c|c|c|c|c|c|}
\hline & \multicolumn{2}{|c|}{$\begin{array}{l}\text { Varones } \\
\text { N: } 55\end{array}$} & \multicolumn{2}{|c|}{$\begin{array}{l}\text { Mujeres } \\
\text { N: } 126\end{array}$} & \multirow[t]{2}{*}{ Z } & \multirow[t]{2}{*}{ Diagnóstico } \\
\hline & $X_{1}$ & $\mathrm{~S}_{1}$ & $X_{2}$ & $\mathrm{~S}_{2}$ & & \\
\hline $\begin{array}{l}\text { Comunicación } \\
\text { interpersonal }\end{array}$ & 55.30 & 8.47 & 56 & 8.40 & .867 & No significativo \\
\hline
\end{tabular}


En la Tabla 4 se observa la comparación de medias en la comunicación interpersonal total entre varones y mujeres de la muestra, mediante la prueba $\mathrm{Z}$. Esta diferencia de medias llevadas a la fórmula $\mathrm{z}$ con dos colas y un nivel de confianza de 0,05 , por consiguiente puede decirse que no existen diferencias estadísticamente significativas entre las medias del grupo de varones con respecto al de mujeres en la comunicación interpersonal.

Tabla 5

Resultados de la comparación de la Comunicación interpersonal según el colegio de procedencia

\begin{tabular}{|c|c|c|c|c|c|c|}
\hline & \multirow{2}{*}{\multicolumn{2}{|c|}{$\begin{array}{l}\text { Estatal } \\
\mathrm{N}: 130\end{array}$}} & \multirow{2}{*}{\multicolumn{2}{|c|}{$\begin{array}{l}\text { Particular } \\
\text { N: } 51\end{array}$}} & \multirow{3}{*}{ Z } & \multirow{3}{*}{ Diagnóstico } \\
\hline & & & & & & \\
\hline & $X_{1}$ & $\mathrm{~S}_{1}$ & $x_{2}$ & $\mathrm{~S}_{2}$ & & \\
\hline Comunicación interpersonal & 55.10 & 8.47 & 56 & 8.40 & .973 & No significativo \\
\hline \multicolumn{7}{|l|}{$\begin{array}{l}* *: \text { Significancia }<0.01 \\
*: \text { Significancia }<0.05\end{array}$} \\
\hline \multicolumn{7}{|c|}{$\begin{array}{l}\text { De acuerdo a la tabla } 5 \text { se observa la comparación de medias en la comunicación } \\
\text { interpersonal entre los alumnos procedentes de colegio estatal y colegio particular } \\
\text { de la muestra, mediante la prueba Z. Esta diferencia de medias llevadas a la fór- } \\
\text { mula z con dos colas y un nivel de confianza de } 0,05 \text {, fue } 0.973 \text { por consiguiente } \\
\text { puede decirse que no existen diferencias estadísticamente significativas entre las } \\
\text { medias del grupo de alumnos procedentes de colegios estatales con respecto al de } \\
\text { los alumnos procedentes colegios particulares en la comunicación interpersonal. }\end{array}$} \\
\hline
\end{tabular}

\section{DISCUSIÓN}

El presente estudio fue aplicado a 181 estudiantes de primer año de la Universidad Nacional Mayor de San Marcos, se obtuvo que el 7.5\% de los sujetos se ubican en el perfil de comunicadores novatos, el $81.5 \%$ se encuentran en el perfil de comunicadores dispuestos y el $11 \%$ se ubican en el perfil de comunicadores naturales, ello implica que en su mayoría se encuentran en un perfil de comunicadores dispuestos, Reyes (1996) describe este perfil como sujetos que al comunicar pueden considerar la actitud que adoptan durante la conversación, el movimiento corporal, los gestos, la entonación y un propósito que lo motiva para realizar el intercambio, con las palabras están expresando sus pensamientos, personalidad y capacidad que tiene para relacionarse con las personas.

Sin embargo, a nivel descriptivo se puede estipular lo siguiente, el $82 \%$ de los evaluados a veces o siempre interrumpen con frecuencia a su interlocutor y prefieren ver TV o leer prensa que dialogar con sus familiares o amigos, el $80 \%$ refiere que siempre o a veces evita la mirada en el hablante, el $73 \%$ a veces o siempre ignora cuál es el momento oportuno para terminar una conversación, el $88 \%$ a veces o siempre se siente nervioso cuando habla frente a personas extrañas, el $90 \%$ a veces o siempre evita las conversaciones y habla con rodeos, el $89 \%$ a veces o siempre interrumpe las conversaciones ajenas, el $84 \%$ a veces o siempre su atención se 
dispersa en una charla, se olvida las palabras y se le dificulta relacionarse con las personas fácilmente, el $85 \%$ a veces o siempre comienza un tema sin terminarlo. Se aprecia que si bien los sujetos cuentan con las capacidades para entablar una comunicación interpersonal, estas no se encuentran desarrolladas, lo que implica que no cuentan con un adecuado interaccionismo simbólico según lo planteado por Mead (1973), ya que no hacen uso adecuado de los símbolos significantes que son la base de la comunicación. Por otro lado, se aprecian dificultades en la comprensión empática propuesta por Kelley y Thibaut (1978), ya que al evitar la mirada en el hablante, evitar las conversaciones y hablar en rodeos, no permite que se genere el nivel de interacción necesaria para el desarrollo de la empatía. Por ende, existen carencias significativas en la comunicación interpersonal en los jóvenes.

En cuanto al análisis de las hipótesis, se aprecia que no existe una diferencia significativa según el género en la comunicación interpersonal de los estudiantes universitarios, en tal sentido, el género no influye en la comunicación interpersonal de un individuo, las variables asociadas a esta estarían más relacionadas al aprendizaje social, tal como lo expone Laborda (2005), quien informa que existe un alto grado de conformidad de los estudiosos en la idea de que las relaciones entre iguales en la infancia contribuyen al desarrollo interpersonal y proporcionan oportunidades únicas para el aprendizaje de habilidades específicas que no pueden lograrse de otro manera ni en otros momentos, asimismo la autora le da importancia a la influencia de las nuevas tecnología que estarían causando un impacto significativo en la comunicación interpersonal del individuo en un futuro próximo.

En cuanto a la segunda hipótesis, se obtuvo que no existe diferencias estadísticamente significativas entre los alumnos procedentes de colegios estatales con respecto a los alumnos procedentes de colegios particulares en la comunicación interpersonal. Ello implica que la pertenencia a un colegio nacional o particular no afecta el desarrollo de las habilidades en la comunicación interpersonal. Sin embargo, es importante resaltar que el colegio al ser un ambiente de interacción importante en el individuo, es donde se debería potencializar el desarrollo de una adecuada comunicación interpersonal, tomando en cuenta lo referido por Rogers (1974), quien considera a la comunicación como una herramienta para la promoción del desarrollo personal, la misma que debe reforzarse desde temprana edad, enseñar a comunicarse.

Se concluye con el presente estudio que el género y la procedencia de un colegio nacional o privado no son determinantes en la comunicación interpersonal de los estudiantes universitarios, asimismo se refuerza la idea del aprendizaje social y de la importancia de la comunicación en la promoción del desarrollo personal. En tal sentido, se recomienda incluir estudios que identifiquen la influencia de las nuevas tecnologías de la comunicación e información en la comunicación interpersonal, las mismas que brindarían pautas en el análisis de los rápidos cambios que se están generando en la actualidad, así como se observa la necesidad de generar estudios comparativos en Latinoamerica, donde el entorno cultural promueve de manera diferencial la comunicación interpersonal. 


\section{CONCLUSIONES}

De acuerdo a las hipótesis planteadas se concluye que:

El género no es una variable que marque diferencias en la comunicación interpersonal de los estudiantes universitarios.

El tipo de colegio no es una variable que marque diferencias en la comunicación interpersonal de los estudiantes universitarios.

\section{REFERENCIAS BIBLIOGRÁFICAS}

Aladro, X. (2009). Espacio interpersonal y sistemas de conducta colectivos. Palabra clave, 12(2), 325-337.

Barbero, M. (1998). De los medios a las mediaciones. Comunicación, cultura y hegemonía. México: Ediciones G. Gili, 300 p.

Berger, C., y Bradac, J. (1995). Interpersonal Comunication. USA: Brace College Publishers.

Garza, M. (2009). Fuentes genealógicas y teóricas esenciales de la comunicación interpersonal. Razón y palabra, (67) Recuperado de http://dialnet.unirioja.es/servlet/ articulo? codigo $=2947209$.

Griffin, E. (2003). A first look at communication theory. USA: MacGraw Hill.

Heider, F. (1958). The psychology of interpersonal relations. New York: Wiley.

Jourard, S. (1971). The Transparent Self. New York: Van Nostrand Reinhold Company.

Kelley, H., y Thibaut, J. (1978). Interpersonal relations. A theory of interdependence. New York: Jhon Wiley y Sons Publication.

Knapp, M., y Vangelisti, A. (1995). Interpersonal comunication. USA: Brace College Publishers.

Laborda, X. (2005). Tecnologías, redes y comunicación interpersonal. Efectos en las formas de la comunicación digital. Anales de documentación, (008), pp. 101-116.

Mead, G. (1973). Espíritu, persona y sociedad. Barcelona: Paidós.

Orozco, G. (1990). Recepción televisiva: tres aproximaciones y una razón para su estudio, Red de Revistas Científicas de América Latina y el Caribe, España y Portugal, IV (14), 319-322.

Rizo, M. (2008). Comunicología y comunicación interpersonal. Revista Comunicologi@: indicios y conjeturas, (9). Recuperado de http://www.robertexto.com/archivo17/ comunicologia.htm

Reyes, G. (1996). El abecé de la pragmática. Madrid: Arco Libros.

Rogers, C. (1974). El proceso de convertirse en persona. Barcelona: Paidós. 